\title{
An Application Based Wireless System for Mobile Ad-hoc Network
}

\author{
Sarvesh Singh Rai \\ Department of C.S.E \\ SGSITS, Indore, (M.P.), India
}

\author{
Dr. W. U. Khan \\ Department of C.S.E \\ SGSITS, Indore, (M.P.), India
}

\author{
Rajesh Ahirwar \\ Department of C.S.E \\ S.A.T.I., Vidisha, (M.P.), India
}

\begin{abstract}
-
Current technologies, such as Bluetooth and IEEE $802.11 \mathrm{~b}$, can form ad hoc networks but is limited in that only single hop networks can be formed. This means that each node can only act as a host, whereas in a multi-hop ad hoc network all nodes act as routers. This thesis concentrated on the problem of adding multi-hop capabilities to existing ad hoc network platforms such as IEEE $802.11 \mathrm{~b}$. This capability will allow a network to be fully dynamic, self-organising and self-configuring. This thesis describes the design and implementation of picoNet - a wireless ad hoc network for mobile handheld devices. As users are increasingly mobile it is more and more common for users to meet and communicate without prior planning and in environments where there is little or no networking infrastructure. Such a network is known as an ad hoc network, where the network is of a dynamic nature without centralised administration. The result was an implementation of the dynamic source routing protocol (DSR) for TCP/IP, for the PCs. The implementation enabled the PCs to form a multi-hop ad hoc network with $802.11 \mathrm{~b}$ wireless cards. A DSR to IP gateway was also implemented and it allowed nodes in the DSR network to access external IP networks. Existing unmodified TCP/IP applications were able to run seamlessly on the picoNet network, providing a useful platform for future extensions.
\end{abstract}

\section{Keywords- MANET, AODV, Multipath Routing.}

\section{INTRODUCTION}

Pervasive computing is computing in an environment where users will be able to access information without going out of their way. To achieve this, the users must be surrounded by technology without knowing so. Pervasive computing is a trend that is currently driving, and will continue to drive many technologies. The vision of picoNet is to create a pervasive network where the underlying technology is invisible and transparent to the user. To achieve this, picoNet will be designed to be compatible with existing networks and networking standards. It will also be designed to work on commonly available hardware and software platforms.
As users are increasingly mobile it is more and more common for users to meet and communicate without prior planning and in environments where there is little or no networking infrastructure. For example, business meetings often require documents to be exchanged and it could happen in a cafe or at the airport. In such situations it is difficult and inconvenient to set up a local area network (LAN) as the network will need to be

created on the fly. Such a network is known as an ad hoc network where the network is of a dynamic nature without centralised administration. Current technologies can form ad hoc networks but is limited in that only single hop networks can be

formed. This means that each node can only act as a host sending directly to the destination. In a multi-hop ad hoc network, all nodes act as routers and neighbouring nodes will forward packets to the final destination. This thesis concentrated on the problem of adding multi-hop capabilities to existing ad hoc network platforms [1] and [3].

An ad hoc network is a network that can be formed without the need for any preexisting networking infrastructure. A mobile ad hoc network (MANET) describes such a network. The IETF MANET Working Group specifies many routing protocols which will allow the formation of mobile ad hoc networks. This thesis describes the design and implementation of picoNet II, a mobile ad hoc network that enables handheld devices to form a dynamic, self-organizing and self-configuring network. To be in line with the picoNet II vision of being compatible with existing networks and networking standards, picoNet II will be designed to be compatible with TCP/IP.

\section{BACKGROUND}

A mobile network consists of mobile devices, herein simply referred to as "nodes", which are free to move about [6]. The way in which mobile networks operate is fundamentally different to traditional fixed networks. In order to understand these differences, and the challenges of designing and implementing a mobile network, some background information needs to be presented. Network models and the concept of routing will be presented first. 
Then the characteristics of mobile ad hoc networks will be compared to fixed wire networks.

\subsection{Network Reference Models}

A computer network is a collection of computers connected by some link which supports data transfer. Designing a computer network to provide various types of connectivity across large numbers of hosts imposes many challenges to the designer. Network reference models help designers deal with these design challenges by abstracting functionality into a layered architecture. Two important network architectures, the OSI reference model and the Internet reference model.

\subsection{The OSI model vs. the Internet Model}

As seen from previous sections, both the OSI model and the Internet model are abstractions of networking functionality. The models differ in the way the abstraction is done, as the Internet model has fewer layers yet describing the same functionality. The OSI model is more general as it can describe any network, but due to many reasons, both technical and non-technical, it was never implemented. The Internet model on the other hand is used widely today. Since most of the networking technology is based on the Internet model, it will be used to define the picoNet system.

\subsection{Mobile Ad Hoc Network (MANET) Characteristics:}

"A "mobile ad hoc network" (MANET) is an autonomous system of mobile routers (and associated hosts) connected by wireless links-the union of which form an arbitrary graph. The routers are free to move randomly and organize themselves arbitrarily; thus, the network's wireless topology may change rapidly and unpredictably. Such a network may operate in a stand alone fashion, or may be connected to the larger Internet." [5] The fundamental difference between fixed networks and MANET is that the computers in a MANET are mobile. Due to the mobility of these nodes, there are some characteristics that are only applicable to MANET. Some of the key characteristics are described below [6]:

1. Dynamic Network Topologies: Nodes are free to move arbitrarily, meaning that the network topology, which is typically multi-hop, may change randomly and rapidly at unpredictable times.

2. Bandwidth constrained links: Wireless links have significantly lower capacity than their hardwired counterparts. They are also less reliable due to the nature of signal propagation.

3. Energy constrained operation: Devices in a mobile network may rely on batteries or other exhaustible means as their power source. For these nodes, the conservation and efficient use of energy may be the most important system design criteria. The MANET characteristics described above imply different assumptions for routing algorithms as the routing protocol must be able to adapt to rapid changes in the network topology. They also present different optimization parameters such as bandwidth overhead and energy usage.

\subsection{Current MANET Research}

Mobile ad hoc networks, or MANET, are fundamentally different to traditional wired networks as wired networks are assumed to be stationary and static. This imposes different design requirement and constraints on routing protocols for MANET. There are two categories of routing protocols: table-driven and on-demand routing. In routing protocols, routing information is periodically advertised to all nodes so all nodes have an up to date view of the network. Alternatively, on-demand routing protocols only discovers a new route when it is required. Hybrid routing protocols also exist and they try to achieve an efficient balance between both categories of protocols.

\subsection{Functional Overview of picoNet}

The function of the picoNet system is to provide multihop capabilities to existing ad hoc networks. For compatibility purposes this functionality should be implemented on the TCP/IP network standard. Any two nodes in the system should be able to communicate across a wireless medium, with end-to-end connectivity achieved by point-to-point packet forwarding at intermediate router nodes [5]. The system should be able to dynamically adapt to node mobility, and nodes entering and leaving the network. Figure 1 shows how packets traverses through the TCP/IP stack in the picoNet system. The multi-hop routing protocol operates at the Internet layer (shaded in grey) of the stack. There are three major sections of the system that needs to be specified and they are routing protocol, hardware and software. The hardware and software specifications will depend on the routing protocol requirements and will also be interdependent.

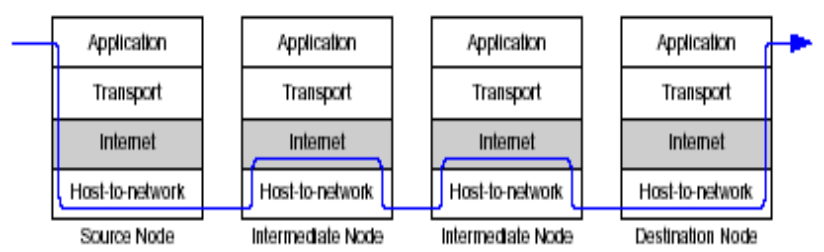

Figure1.: Network stack traversal in picoNet 


\begin{tabular}{|c|}
\hline Application (Telnet, FTP, DNS) \\
\hline Transport (TCP, UDP) \\
\hline Internet (IP) \\
\hline Host-to-network (802.11, Bluetooth) \\
\hline
\end{tabular}

Figure 2: TCP/IP stack

\subsection{Routing Protocol Requirements}

The function of the routing protocol is to provide multihop capabilities. The routing protocol and its implementation must be fully compatible with existing TCP/IP networks. To achieve this compatibility, the routing protocol will need to operate in the Internet layer maintaining compatibility with the transport layer and the host-to-network layer. Figure 2 shows this structure with the Internet layer shaded in grey. This compatibility will allow existing applications, transport protocols and network interfaces to operate without modifications. There are some key requirements in mobile ad hoc networks (MANET) and they are listed below [6]:

- Distributed operation: This is an obvious and necessary property of MANET.

- Loop-freedom: Not a required property but desirable for any routing protocol, as it avoids packets spinning around in the network causing performance to degrade.

- On demand operation: Instead of maintaining routing information between all nodes at all times, the routing information is generated on a demand or need basis. Although it may increase the route discovery delay, it utilises network energy and bandwidth resources more efficiently. This efficiency is especially important for mobile devices where bandwidth and energy resources are limited.

There are also some performance measures of ad hoc routing protocols and they are described below:

- Route acquisition time: A measure of the time taken to discover a new route.

- Packet data overhead: The amount of extra data exchanged for ad hoc routing to operate.

- Packet overhead: The number of extra packets sent by the ad hoc routing protocol. These performance measures ultimately affect the end-to-end delay experienced by users. The routing protocol should be selected to be meet the requirements of MANET with minimized end-to-end delay.

\section{PROPOSED TECHNOLOGY}

Ad hoc On-demand Distance Vector Routing (AODV) is an on-demand version of the table-driven Dynamic Destination-Sequenced Distance-Vector (DSDV) protocol [6]. To find a route to the destination, the source broadcasts a route request packet. This broadcast message propagates through the network until it reaches an intermediate node that has recent route information about the destination or until it reaches the destination. When intermediate nodes forwards the route request packet it records in its own tables which node the route request came from. This information is used to form the reply path for the route reply packet as AODV uses only symmetric links. As the route reply packet traverses back to the source, the nodes along the reverse path enter the routing information into their tables. Whenever a link failure occurs, the source is notified and a route discovery can be requested again if needed.

The Temporally Ordered Routing Algorithm (TORA) is a highly adaptive, efficient and scalable routing algorithm. It is a source-initiated on-demand protocol and it finds multiple routes between the source and the destination. TORA is a fairly complicated protocol but its main feature is that when a link fails the control messages only propagates around the point of failure. While other protocols need to re-initiate a route discovery when a link fails, TORA would be able to patch itself up around the point of failure. This feature allows TORA to scale up to larger networks but has higher overhead for smaller networks.

The Dynamic Source Routing (DSR) protocol is a sourcerouted on-demand protocol. There are two major phases for the protocol: route discovery and route maintenance. The key difference between DSR and other protocols is the routing information is contained in the packet header. Since the routing information is contained in the packet header, the intermediate nodes do not need to maintain routing information. An intermediate node may wish to record the routing information in its tables to improve performance, but this is not mandatory. Another feature of DSR is that it supports asymmetric links as a route reply can be piggybacked onto a new route request packet. DSR is suited for small to medium sized networks as its packet overhead (not packet data overhead) can scale all the way down to zero when all nodes are relatively stationary. The packet data overhead will increase significantly for networks with larger hop diameters as more routing information will need to be contained in the packet headers.

\subsection{Protocol Selection}

Out of all the routing protocols, TORA was the most complex and also the most scalable. These properties of 
TORA may be ideal for an ad hoc routing protocol, but it was not preferred for this thesis as ease of implementation was one of the key selection factors. The main difference between AODV and DSR was the way the routing information was exchanged. In AODV the information was stored at each node where as in DSR the routing information was included in each packet. Simulation results have shown that AODV and DSR have similar performance with DSR being more efficient with higher node mobility and AODV more efficient at lower node mobility.

\subsection{Route Discovery}

Route discovery is the process in which a source node discovers a route through the network to some arbitrary destination node. Every node has a route cache which contains recent routes to other nodes on the network. If a node needs to send information to some destination and a route is found in the route cache then the node will use that route. Otherwise the source node will initiate a route discovery process by sending a route request packet across the network. Figure 3 illustrates the route discovery process and the propagation of route request packets. Every route request packet has a unique identification number. Nodes cache this identification number and discards subsequent route request packets with the same identification number. In the example shown in Figure 3, node $\mathrm{D}$ received the route request from node $\mathrm{C}$ first and it discarded the route request from node $B$. As the route request propagates through each node, each node adds its own address to the route request if it is not already present. This ensures loop-free routes. When the route request reaches the final destination, a route reply packet is returned to the source node from the destination node. For asymmetric links the route reply may be

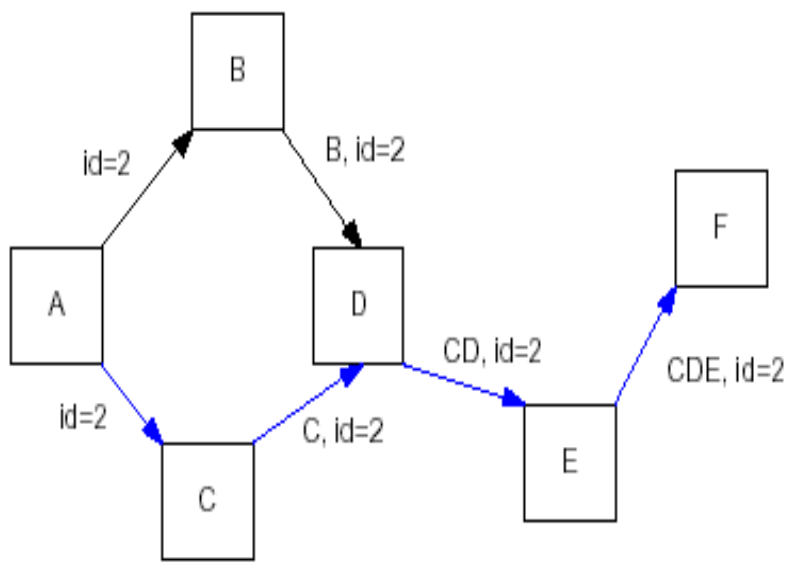

Figure 3: Route Discovery Process piggybacked onto a new route request. In our system where $802.11 \mathrm{~b}$ links are bidirectional, the route reply will simply contain the route recorded in the route request packet in reverse order. In the event that the final destination is not present or completely out of range, route requests will be resent by the source node after a timeout which backs off exponentially.

\subsection{Packet Forwarding-}

When a node wishes to send packets and has a route to the destination, it adds the full source route to the each of the packets. Along with the source route the number of segments left is also added and source node will initialize this number to the length of the source route. The number of segments left is the number of hops left for the packet to reach its destination and it gets decremented each hop. It is used for intermediate nodes to index the next hop address from the source route so the packet can be forwarded to the next node. The packet forwarding process is illustrated in Figure 4.

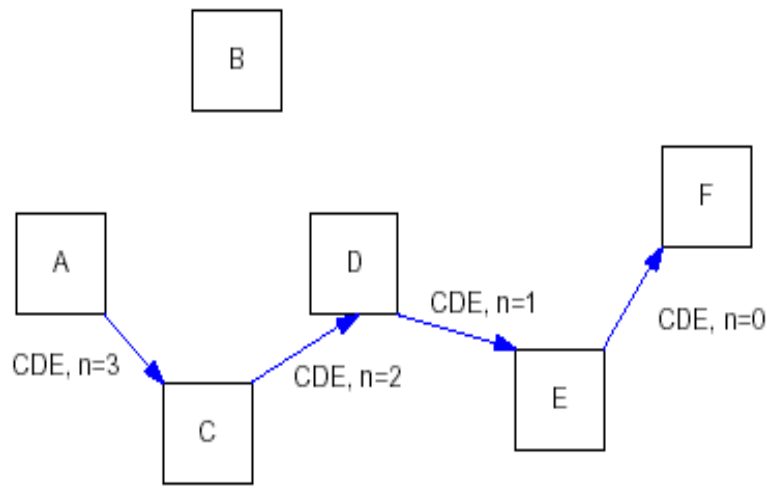

Figure 4: Packet Forwarding Process

\subsection{Route Maintenance}

As nodes in a MANET move around, links will be down and routes need to be maintained. This is called route maintenance. During packet forwarding every node is responsible confirming that the packet was received by the next hop. There are three ways to get this acknowledgement and they are listed below:

- MAC layer acknowledgement: this is supplied by the underlying MAC layer and technologies like IEEE 802.11 b support it.

- Passive acknowledgement: this confirmation comes from nodes overhearing the next node forwarding the packet. It can be used on every hop except the last hop. This requires the network interfaces to work in promiscuous mode so it can overhear packets sent to other nodes. 
- Network layer acknowledgement: this is when the node explicitly requests a DSR specific acknowledgement to be returned by the next hop as it was the easiest to implement. MAC layer acknowledgements require interfacing the routing code with the network interface driver which would introduce unnecessary work.

Passive acknowledgements were not feasible as there was little support for promiscuous mode from the network drivers. When no acknowledgement has been received by the node sending a packet after a set timeout, the packet is resent after a timeout a set number of times. If no acknowledgement is received after the retransmission, then a route error packet will be sent back to the source node to indicate that the link is broken. In the example shown in Figure 5, the link from node D to node $\mathrm{E}$ is broken so node $\mathrm{D}$ will send a route error packet back to node A indicating that link D-E is broken. Upon receipt the route error packet, the source node will update the route cache accordingly. The source node will use another route if it is present in the route cache; otherwise a new route discovery process is initiated.

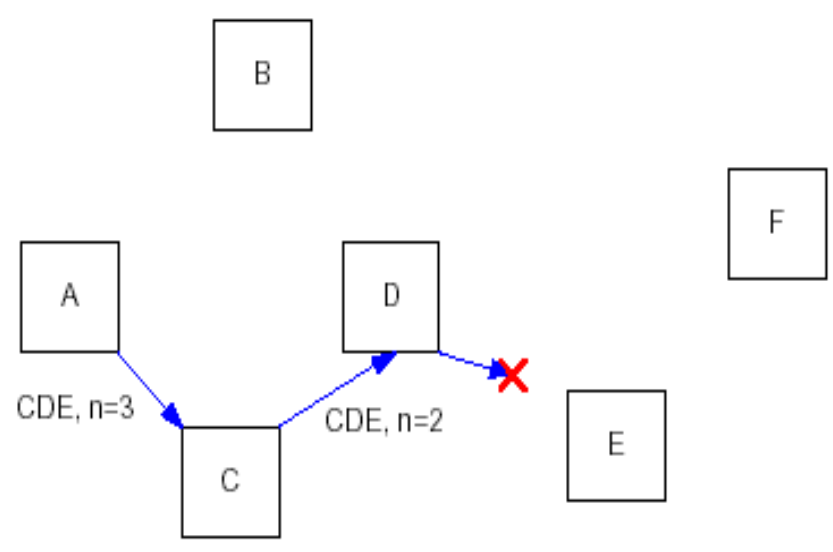

Figure 5: Route Maintenance Process

\subsection{Packet Formats}

The Dynamic Source Routing protocol makes use of a special header, which carries control information that can be included in any IP packet. The DSR header in a packet contains a fixed sized 4 byte section which is followed by a sequence of zero or more DSR options carrying optional information. The total length of the DSR options is stored in the DSR header. The DSR header is inserted in the packet following the IP header and before any transport layer information. Figure 5 illustrates this. The format of the IP header will not be modified but some fields in the IP header will need to be changed to differentiate a DSR packet from a normal IP packet. Figure 6 shows the IP header with the modified fields shaded in grey. The protocol field is changed to a unique number indicating that the packet is a DSR packet. As DSR information is inserted to the packet, the total length of the packet must also be changed. The destination is changed to a broadcast address for route request packets and when any field in the IP header changes, the checksum must be recalculated. The fixed portion of the DSR header shown in Figure 7 and it contains three fields of which two are currently used. The next header field is used to record the IP protocol

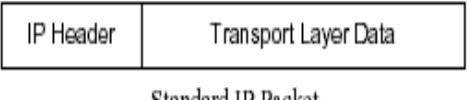

Standard IP Packet

\begin{tabular}{|l|l|l|l|}
\hline IP Header & DSR Header & DSR Options & Transport Layer Data \\
\hline
\end{tabular}

IP Packet with DSR information

Figure 5.4.: DSR and IP packet structure

\begin{tabular}{|c|c|c|c|c|c|}
\hline Version & IHL & Type of Service & \multicolumn{2}{c|}{ Total Length } \\
\hline \multicolumn{2}{|c|}{ Identification } & $\begin{array}{c}\text { D } \\
\text { F }\end{array}$ & Fragment Offset \\
\hline Time To Live & Protocol & Header Checksum \\
\hline \multicolumn{2}{|c|}{ Source Address } \\
\hline
\end{tabular}

Figure 6: IP Header Format

number of the original packet, so when the DSR information is removed at the final destination, the original packet can be constructed. This method is completely transparent to the upper protocol layers. The payload length field defines the total length of all the DSR options carried in this packet. Every DSR option has an option type field and an option length field. The option type field indicates the type of the option which will determine the format of the option. The option length field indicates the size of the option. Figure 8 shows and example of a DSR option, the route request option. The option type and option length fields are shaded in grey.

\begin{tabular}{|l|l|l|}
\hline NextHeader & Reserved & Payload Length \\
\hline
\end{tabular}

Figure 7: Fixed Portion of the DSR Header 


\begin{tabular}{|c|c|c|}
\hline Option Type & Opt Data Len & Identification \\
\hline \multicolumn{3}{|c|}{ Target Address } \\
\hline Address[1] \\
\hline$\ldots$ \\
\hline Address[n]
\end{tabular}

Figure 8: Route Request Option Format

\subsection{Protocol Optimisations}

Many routing optimisations were outlined in the DSR Internet-draft, but only a few basic optimisations were implemented due to time constraints, and that optimisations were not necessary for the demonstration of the picoNet proof-of-concept. Many optimizations also required the wireless links to be in promiscuous mode so nodes can cache and process overheard information. The few basic optimisations that were implemented involved nodes caching routes from packets they received or forwarded. This occurs whenever a node propagates a route request or a route error, and also occurs when a node forwards a packet as they contain route information. These optimisations reduce the number of route discoveries initiated.

\subsection{Protocol Modifications}

There were two modifications made to the DSR protocol. The first one changed the way route discoveries were made to nodes outside of a DSR network. The second change was an extension to the protocol, dealing with IP packet fragmentation. Both of these changes are detailed below.

\section{- External Node Route Discovery}

The specified method for conducting a route discovery to external nodes (nodes outside the DSR network) was to initiate a normal route request with the route reply indicating the last hop is external. This meant that a route entry was required for every external destination. In conventional network setups, there is usually one gateway machine per subnet to route between the subnet and external networks. This subnet idea has been applied to the DSR implementation and means that nodes would simply initiate a route discovery to the gateway machine if the destined node is external to the DSR network. This results in one route entry for all external nodes. The subnet method is less flexible than the method specified in the draft, as all DSR nodes have to be within the same subnet and only one gateway can exist, but it is easier to implement.

\section{- IP Packet Fragmentation}

During a packet's traversal through a network it may go through different MAC technologies with different maximum frame sizes. IP fragmentation deals with this by splitting packets which are too big, into smaller fragments and reassembling them again when the destinations is reached. The current draft for the DSR protocol does not support packet fragmentation. In order to demonstrate compatibility with existing applications, fragmentation is needed. Thus fragmentation support was added to the DSR protocol. This extension involved duplicating the DSR information during packet fragmentation and requesting a separate network layer acknowledgement for each fragment.

\section{RESULTS}

Qualitative analysis is provided here as quantitative analysis can be difficult. The difficulty comes from the fact that the analysis depends on many factors such as user movement and network usage, not mentioning environmental effects on wireless links. Quantitative performance analysis is more suited to network simulations, which is beyond the scope of this thesis.

Performance problems were observed with TCP connections, as standard TCP is not wireless aware. TCP assumes that lost packets are caused by congestion, and will slow down unnecessarily when packets have been lost due to the wireless environment. This slow down occurs when packets are lost during a TCP connection, which can be caused by users temporarily moving out of range. The slow down is quite severe and very noticeable to the user. This problem is caused by TCP, not DSR and users can reopen the TCP connection to get around this problem. A proper solution would be to have a wireless aware TCP, which is an area of research that is beyond the scope of this thesis.

\section{CONCLUSION AND FUTURE WORK}

PicoNet was designed to provide multi-hop capabilities to existing ad hoc networks. The system was able to create a dynamic, self-organising, and self-configuring network on the fly without the aid of any networking infrastructure. This thesis has demonstrated that it is possible to create extensions to existing technologies transparently, maintaining full compatibility. The implementation was fully functional and can be used in many real world applications. The current design presents more useful platform for future extensions, and may take us closer to realizing the vision of pervasive computing, where technology blends seamlessly with everyday life. 


\section{REFERENCES}

[1] Zahian Ismail and Rosilah Hassan, "Ad Hoc Network Performance of AODV Routing Protocol in Mobile", IEEE2010.

[2] Pore Ghee Lye and John C. McEachen," A Comparison of Optimized Link State Routing with Traditional Routing Protocols in Marine Wireless Ad-hoc and Sensor Networks", 40th Hawaii International Conference on System Sciences - IEEE2007

[3] Fahimeh Rookhosh, Abolfazl Toroghi Haghighat, Saeed Nickmanesh," Disjoint Categories in Low Delay and On-Demand Multipath Dynamic Source Routing Adhoc Networks", IEEE2008

[4] Carlos de M. Cordeiro and Dharma P. Agrawal," Employing Dynamic Segmentation for Effective COlocated Coexistence between Bluetooth and IEEE 802.11 WLANs", IEEE 2002.

[5] Andrew Zhang, David B. Smith, Dino Miniutti, Leif W. Hanlen ,David Rodda, Ben Gilbert," Performance of Piconet Co-existence Schemes in Wireless Body Area Networks", IEEE Communications Society subject matter experts for publication in the WCNC 2010 proceedings.
[6 ]S. Corson and J. Macker, "Mobile ad hoc networking (manet): Routing protocol performance issues and evaluation considerations." http://www.ietf.org/rfc/ rfc2501.txt, January 1999.

[7] IETF, "Mobile ad-hoc networks (manet)." http://www.ietf.org/html.charters/manet-charter.html, April 2001.

[8] D. B. Johnson and D. A. Maltz, "Dynamic source routing in ad hoc wireless networks," in Mobile Computing (T. Imielinski and H. Korth, eds.), ch. 5, pp. 153-181, Kluwer Academic Publishers, 1996.

[9] J. Broch, D. A. Maltz, D. B. Johnson, Y.-C. Hu, and J. Jetcheva, "A performance comparison of multi-hop wireless ad hoc network routing protocols," in Proceedings of the Fourth Annual ACM/IEEE International Conference on Mobile Computing and Networking, (Dallas, Texas), ACM, October 1998.

[10] D. B. Johnson, D. A. Maltz, and Y.-C. Hu, "The dynamic source routing protocol for mobile ad hoc networks." http://www.ietf.org/internet-drafts/ draft-ietfmanet-dsr-05.txt, March 2001. Work in progress. 